\title{
Effect of Vibration Training on Anaerobic Power and Quardroceps Surface EMG in Long Jumpers
}

\author{
Bin Liu $^{1}$, Jiong Luo ${ }^{1}$ \\ ${ }^{1}$ College of Physical Education, Southwest University, Beibei, Chongqing, 400715, China \\ Correspondence: Bin Liu, College of Physical Education, Southwest University, Beibei, Chongqing, 400715, China
}

Received: May 1, 2015 Accepted: May 12, $2015 \quad$ Online Published: May 19, 2015

doi:10.11114/jets.v3i4.807 URL: http://dx.doi.org/10.11114/jets.v3i4.807

\begin{abstract}
Objective: To explore the anaerobic power and surface EMG (sEMG) of quardrocep muscle in lower extremities after single vibration training intervention. Methods: 8 excellent male long jumpers voluntarily participated in this study. Four intervention modes were devised, including high frequency high amplitude (HFHA,30Hz,6mm), low frequency low amplitude(LFLA, 15Hz,3mm), high frequency low amplitude (HFLA,30Hz,3mm), and non-vibration (CON). All subjects received a knee extensor maximal voluntary contraction (MVC) test before the vibration. After the test, a $5 \times 1$ min. vibration would be carried out and the subjects were asked to pedal. Results: Peak power and average power output of the four vibration treatment methods did not reach obvious level. There was no obvious difference in root-mean-square(RMS) EMG of the dominant leg among the four treatments. The RMS EMG of the non-dominant leg of HFLA method during peak power output was much higher than that of HFHA and CON. The quotient between root-mean-square and peak power of the four groups did not reach significant level. Conclusion: Single vibration could not improve the anaerobic performance of long jumpers and HFLA might decrease the work efficiency of the non-dominant leg muscle of excellent long jumpers participating anaerobic test.
\end{abstract}

Keywords: EMG, Vibration training, long jumper, anaerobic power

\section{Introduction}

Muscle force and explosive power matter in any sport event. How to improve muscle force and explosive power has become the concern of many trainers and players. Many researchers and trainers have been making great efforts to explore new training methods, expecting to enhance players' competitiveness. Vibration training uses mechanical method to vibrate muscle so as to stimulate neuromuscular system. Its intensity depends on the frequency and amplitude of vibration instrument. The physiological mechanism of such method is that the stimulation caused by vibration can regulate neuromuscular system through monosynaptic and polysynaptic path and thus improve muscle activity. There are two applications of the whole-body vibration training in improving muscle force and explosive power. The first is to apply direct stimulation by placing vibrator on muscle belly or tendon; and the second is to apply indirect stimulation through the vibration of vibration platform.

Single whole-body vibration can improve muscle performance. Cochrance (2005) studied the influence of single whole-body vibration on swing-arm jumping squat and flexibility. The result showed that the vibration could improve flexibility and increase the height of swing-arm jumping squat. The study of Cormie (2006) showed that single whole-body vibration (frequency $30 \mathrm{~Hz}$, amplitude $2.50 \mathrm{~mm}$ ) could significantly increase the height of jumping squat and prolong the duration of half-squat subjects on vibration platform. Bosco (1999a) pointed out that whole-body vibration could improve the muscle force of lower limbs and arm flexor rapidly and markedly. The study of Issurin (1999) showed that vibration can markedly increase the maximum power when elbow did centered motion. Such increase in professional players could be as much as $10.4 \%$, better than that in amateurs (increase by about $7.9 \%$ ). Thus it is obvious that single whole-body vibration can improve the anaerobic exercise performance of players. Lower limbs explosive force training is vital for long jumpers. However, there is no empirical study exploring the influence of single whole-body vibration on the anaerobic exercise performance of long jumpers. Thus this paper bears practical significance. 


\section{Subjects and Methods}

\subsection{Subjects}

8 long jumpers (Athletic performance: nation level 2; body height: $179.4 \pm 4.36 \mathrm{~cm}$; body weight:76.2 $\pm 3.55 \mathrm{~kg}$; athletic years: $8.3 \pm 1.5 \mathrm{yrs}$ ) from the track and field team of Southwest University voluntarily participated in this study. The study was scheduled in the special preparation period of annual training plan, namely 4 weeks before regulation game. Before the test, we let every subjects understand the purpose of this experiment and get their and their trainer's approval. All subjects were required to avoid doing strenuous exercise and eating foods containing alcohol and caffeine 24 hours before the test.

\subsection{Methods}

\subsubsection{MVC Test}

Before the whole-body vibration, all subjects should go through a MVC test on vastus lateralis muscle, vastus medialis muscle and rectus femoris of both feet. The test starts after 10min warm-up running. Each foot goes through 3 tests. Each test lasted for $5 \mathrm{~s}$ with an interval of $60 \mathrm{~s}$ between tests. The subjects sat on test platform with their legs falling naturally along the edge and the knee-joint range being 90-125 degree. The subjects raised their legs as possible as they could while the researcher pressed hard their tibialis anterior muscle and ankle with both hands so as to maintain the maximum isometric contraction of quadriceps femoris. At the same time, myoelectricity test system gathered the vastus lateralis muscle, vastus medialis muscle and rectus femoris EMG.

\subsubsection{Vibration Training Design}

1-2 min after the MVC test, subjects were asked to half squat on the vibration platform (knee angle is $100^{\circ}$ and hip angle is $90^{\circ}$ ) to undergo five times whole-body vibration. Each vibration lasted for $1 \mathrm{~min}$ with an interval of $1 \mathrm{~min}$ between vibrations. Four vibration treatments (HFHA, LFLA, HFLA, CON) would be carried out in this study with an interval of 4 days between treatments to allay fatigue and eliminate the effect of relevant stimulation.

\subsubsection{Anaerobic Power and Myoelectricity Test on Lower Limbs}

After the $5 \times 1$ min vibration, subjects were asked to go through a 30s Wingate anaerobic power test on a Monark-834 cycle ergometer (made in Sweden) immediately. Before test, seat height and pedal of the ergometer are adjusted and the subjects were allowed to adapt to the load they are about to bear (resistance load $=0.08 \times$ body weight). As soon as the test starts, the subjects should pedal as fast as they can for 30s. During the process, verbal encouragement was given till the end of the test. Calculation method of power: power $(\mathrm{w})=$ resistance load $(\mathrm{kg}) \times$ circles $\times 11.765$. Each six $5 \mathrm{~s}$ was deemed as a group. The maximum anaerobic capacity was the $5 \mathrm{~s}$ with the most circles. Generally, it was the first $5 \mathrm{~s}$ which could reflect ATP and CP decomposition energy supply. Average anaerobic capacity is the mean wattage value of the six 5s, reflecting non-lactic acid and lactic acid decomposition energy supply. Anaerobic capacity descent rate, also called fatigue index, was (the maximum anaerobic capacity value - the minimum anaerobic capacity value) $\div$ (the maximum anaerobic capacity value) $\times 100$. TB-0810 (8-channel wireless surface EMG, China) was used to monitor the myoelectricity of the vastus lateralis muscle, vastus medialis muscle and rectus femoris of both legs.

Synchronizing gear was made up of wireless synchronizer of the surface electromyograph and luminous diode. The experimenter triggers synchronizing remote control while the surface electromyograph starts collecting data and the diode synchronizes video camera and the surface electromyograph. The principal optic axis of a JVC9800 high-speed camera whose photo frequency was $250 \mathrm{frame} / \mathrm{s}$ and time of exposure was $1 / 250 \mathrm{~s}$ directly faces the hip joint of the subjects, with the shooting distance of $6 \mathrm{~m}$. Purpose of the synchronization is to find out the corresponding EMG value of quadriceps femoris at the moment of lower limbs peak power output.

\subsubsection{Statistical Method}

\section{1) Calculation of EMG Value}

Sampling frequency of surface electromyograph was set at $1000 \mathrm{~Hz}$. Test analysis software was used to conduct $20 \mathrm{hz}$ filtering operation on the original EMG, then conduct full-wave rectification on the EMG and calculate EMGrms amplitude. Took the EMGrms of MVC as reference and gave standard treatment to the peak-power-corresponding EMGrms during the 30s Wingate anaerobic capacity test to get the standard EMGrms percentage of dominant leg and non-dominant leg. The calculation method of the total EMG value of quadriceps femoris was to add the EMGrms of vastus lateralis muscle, vastus medialis muscle and rectus femoris and then divide the MVC EMGrms of the three muscle groups.

\section{2) Calculation of Neural Efficiency}

After the 30sWingate anaerobic capacity test, used camera to review and analyze the average power of the long jumpers, 
find out the EMGrms of astus lateralis muscle, vastus medialis muscle and rectus femoris corresponding with peak power output, calculated the mean EMG value of EMGrms, and then converted the EMGrms into power/body weight $(\mathrm{kg})$.

\section{3) Statistic Analysis}

SPSS 13.0 was used in this study.repeated measure one-way ANOVA and LSD multiple comparison among the four intervention modes. Significant level of all parameters was set at $a=0.05$.

\section{Results}

\subsection{Analysis on Anaerobic Capacity Features of the Four Methods}

1) Whether for the dominant leg (the leg to jump) or non-dominant leg, there was no obvious difference in peak power (W) and relative peak power $(\mathrm{W} / \mathrm{kg})$ among the four methods. But the peak power and relative peak power of the dominant leg were superior to the non-dominant leg in all of the four treatments.

2) There was no obvious difference in the power descent rate of dominant leg and non-dominant leg among the four methods. But the descent rate of the non-dominant leg was much higher than that of the dominant leg in all of the four treatments.

3) There was obvious difference in the time to reach maximum power (s) among the four methods. HFHA (a) needed the least time to reach peak power; then was the HFLA (c); LFLA (b) was the third; and CON (d) was the last. When it came to the comparison between the dominant leg and the non-dominant leg, the time needed to reach maximum power for the dominant leg is much shorter than the non-dominant leg under HFHA (a) and HFLA (c) while the other two methods had no difference for the two legs.

Table 1. Anaerobic metabolism capacity comparison between different vibration training modes in lower extremities

\begin{tabular}{|c|c|c|c|c|c|}
\hline & & Peak power $(\mathrm{W})$ & $\begin{array}{ll}\text { Relative } & \text { peak } \\
\text { work(W/kg) }\end{array}$ & Power decline $\%$ & $\begin{array}{l}\text { Time to maximal } \\
\text { power }(\mathrm{s})\end{array}$ \\
\hline \multirow[t]{2}{*}{ HFHA(a) } & Dominant & $951.46 \pm 121.65^{*}$ & $17.02 \pm 2.45^{*}$ & $14.74 \pm 4.52$ & $5.54 \pm 1.69$ \\
\hline & Non-dominant & $921.31 \pm 103.33$ & $15.56 \pm 3.45$ & $16.29 \pm 3.69 *$ & $6.21 \pm 2.77 *$ \\
\hline \multirow[t]{2}{*}{ LFLA(b) } & Dominant & $943.17 \pm 104.82 *$ & $16.41 \pm 2.44 *$ & $15.05 \pm 3.27$ & $7.39 \pm 3.12$ \\
\hline & Non-dominant & $923.45 \pm 125.64$ & $15.11 \pm 1.56$ & $16.11 \pm 4.11 *$ & $7.14 \pm 2.29$ \\
\hline \multirow[t]{2}{*}{$\operatorname{HFLA}(\mathrm{c})$} & Dominant & $948.65 \pm 141.14 *$ & $16.51 \pm 2.01 *$ & $14.89 \pm 3.24$ & $6.35 \pm 3.28$ \\
\hline & Non-dominant & $921.35 \pm 123.15$ & $15.31 \pm 1.29$ & $16.02 \pm 3.42 *$ & $7.15 \pm 1.98 *$ \\
\hline \multirow[t]{2}{*}{$\mathrm{CON}(\mathrm{d})$} & Dominant & $948.23 \pm 131.43^{*}$ & $16.55 \pm 2.17 *$ & $15.11 \pm 4.58$ & $7.15 \pm 2.56$ \\
\hline & Non-dominant & $915.56 \pm 138.69$ & $15.25 \pm 1.78$ & $15.58 \pm 3.37 *$ & $8.03 \pm 2.26$ \\
\hline \multirow{2}{*}{ LSD } & between dominants & $\begin{array}{l}\text { Pab, Pac, Pad, Pbc, } \\
\text { Pbd, Pcd }\end{array}$ & $\begin{array}{l}\text { Pab, Pac, Pad, } \\
\text { Pbc, Pbd, Pcd }\end{array}$ & $\begin{array}{l}\text { Pab, Pac, Pad, Pbc, } \\
\text { Pbd, Pcd }\end{array}$ & $\begin{array}{l}\mathrm{Pab}^{*}, \mathrm{Pac}^{*}, \\
\mathrm{Pbc}^{*}, \mathrm{Pbd}^{*}, \mathrm{Pcd}^{*}\end{array}$ \\
\hline & $\begin{array}{l}\text { between } \\
\text { Non-Dominants }\end{array}$ & $\begin{array}{l}\text { Pab, Pac, Pad, Pbc, } \\
\text { Pbd, Pcd }\end{array}$ & $\begin{array}{l}\text { Pab,Pac,Pad Pbc, } \\
\text { Pbd, Pcd }\end{array}$ & $\begin{array}{l}\mathrm{Pab}^{*}, \mathrm{Pac}^{*}, \mathrm{Pad}^{*}, \\
\mathrm{Pbc}^{*}, \mathrm{Pbd}^{*}, \mathrm{Pcd}^{*}\end{array}$ & $\begin{array}{l}\mathrm{Pab}^{*}, \mathrm{Pac}^{*}, \mathrm{Pad}^{*} \\
\mathrm{Pbc}^{*}, \mathrm{Pbd}^{*}, \mathrm{Pcd}^{*}\end{array}$ \\
\hline
\end{tabular}

Note: * stands for the significant difference between dominant and Non -dominant of the participants, Pab* stands for the significant difference between HFHA and LFLA in different vibration modes in LSD multiple comparison. The mark in the below has the same meaning.

\subsection{Analysis on the Surface Myoelectric Activity of Quadriceps Femoris}

1) There was no difference in the EMGrms\% of rectus femoris of the dominant leg among the four methods. While for the non-dominant leg, electro-discharge was the highest $(83.10 \pm 25.14)$ under LFLA (b); then was HFHA (a) and HFLA (c); and CON (d) was the last. Except the CON (d), the rectus femoris activation level of the non-dominant leg was higher than the dominant leg in other three methods.

2) Vastus medialis EMGrms\% of the dominant leg had the strongest activity under HFHA (a) and LFLA (b), much higher than that of HFLA (c) and CON (d). On the contrary, HFLA (c) and CON (d) the strongest activity level for non-dominant leg, much higher than the other two. The vastus medialis activation level of the dominant leg was much higher than that of the non-dominant leg under LFLA (b) while the opposite was true under HFLA (c) and CON (d).

3) Vastus lateralis EMGrms\% of the dominant leg had the strongest activity under LFLA (b) and HFLA (c), much higher than that of HFHA (a) and CON (d). For the non-dominant leg, the activity was the strongest under HFLA (c), much higher than the other three. The vastus lateralis activation level of the dominant leg was much higher than that of the non-dominant leg under LFLA (b) and CON (d) while there was no difference between the dominant and 
non-dominant legs under the other two.

4) Quadriceps femoris EMGrms\% of the dominant leg had the strongest activity $(81.47 \pm 16.01)$ under HFLA (c), much higher than the other three methods. For the non-dominant leg, the activity was the strongest under LFLA (b) and HFLA (c), much higher than the other two methods. The quadriceps femoris activation level of the dominant leg was much higher than that of the non-dominant leg under LFLA (b) and HFLA (c) while there was no difference between the dominant and non-dominant legs under the other two.

Table 2. sEMG characteristics comparison between different vibration training mode of quadriceps muscle (EMGrms\%)

\begin{tabular}{|c|c|c|c|c|c|}
\hline & & Rectus femoris & Vastus intermedius & Vastus lateralis & Quadriceps \\
\hline \multirow[t]{2}{*}{ HFHA(a) } & Dominant & $64.98 \pm 17.13$ & $97.12 \pm 34.11 *$ & $83.14 \pm 15.56$ & $75.89 \pm 15.57$ \\
\hline & Non-dominant & $76.69 \pm 17.25^{*}$ & $82.17 \pm 22.54$ & $81.25 \pm 15.66$ & $74.58 \pm 17.55$ \\
\hline \multirow[t]{2}{*}{ LFLA(b) } & Dominant & $63.36 \pm 19.15$ & $95.45 \pm 33.65 *$ & $94.57 \pm 27.12 *$ & $76.58 \pm 15.26$ \\
\hline & Non-dominant & $83.10 \pm 25.14 *$ & $88.17 \pm 26.38$ & $82.25 \pm 22.36$ & $85.69 \pm 16.35^{*}$ \\
\hline \multirow[t]{2}{*}{ HFLA(c) } & Dominant & $66.25 \pm 14.55$ & $82.58 \pm 28.74$ & $95.78 \pm 26.39$ & $81.47 \pm 16.01$ \\
\hline & Non-dominant & $75.15 \pm 20.12 *$ & $94.58 \pm 30.14 *$ & $95.68 \pm 24.76$ & $87.77 \pm 19.51 *$ \\
\hline \multirow[t]{2}{*}{$\mathrm{CON}(\mathrm{d})$} & Dominant & $67.12 \pm 18.19$ & $81.45 \pm 17.58$ & $84.57 \pm 28.45^{*}$ & $74.26 \pm 13.67$ \\
\hline & Non-dominant & $69.17 \pm 21.36$ & $95.12 \pm 36.58 *$ & $78.59 \pm 15.34$ & $78.32 \pm 15.47$ \\
\hline \multirow{2}{*}{ LSD } & $\begin{array}{l}\text { Between } \\
\text { dominants }\end{array}$ & $\begin{array}{l}\text { Pab, Pac, Pad, Pbc, } \\
\text { Pbd ,Pcd }\end{array}$ & $\begin{array}{l}\mathrm{Pab} \mathrm{Pac}^{*} \mathrm{Pad}^{*} \\
\mathrm{Pbc} * \mathrm{Pbd}^{*} \mathrm{Pcd}\end{array}$ & $\begin{array}{l}\mathrm{Pab}^{*}, \mathrm{Pac}^{*}, \quad \mathrm{Pad}, \\
\mathrm{Pbc}, \mathrm{Pbd}^{*}, \mathrm{Pcd}^{*}\end{array}$ & $\begin{array}{l}\mathrm{Pab}^{*}, \mathrm{Pac}^{*}, \mathrm{Pad}^{*}, \\
\mathrm{Pbc}^{*}, \mathrm{Pbd}^{*}, \mathrm{Pcd}^{*}\end{array}$ \\
\hline & $\begin{array}{l}\text { Between } \\
\text { non-dominants }\end{array}$ & $\begin{array}{l}\text { Pab*, Pac, } \quad \text { Pad* }^{*}, \\
\text { Pbc*, Pbd*, Pcd*, }\end{array}$ & $\begin{array}{l}\text { Pab, Pac, Pad, Pbc, } \\
\text { Pbd, Pcd }\end{array}$ & $\begin{array}{l}\mathrm{Pab}, \mathrm{Pac}^{*}, \quad \mathrm{Pad}, \\
\mathrm{Pbc} *, \mathrm{Pbd}, \mathrm{Pcd}^{*}\end{array}$ & $\begin{array}{l}\mathrm{Pab}^{*}, \mathrm{Pac}^{*}, \mathrm{Pad}^{*}, \\
\mathrm{Pbc}^{*}, \mathrm{Pbd}^{*}, \mathrm{Pcd}^{*}\end{array}$ \\
\hline
\end{tabular}

3.3 Analysis on the Neuron Efficiency of Quadriceps Femoris of the Four Methods

1) Whether for the dominant leg or non-dominant leg, there was no obvious difference in the neuron efficiency of rectus femoris, vastus medialis muscle, vastus lateralis muscle or quadriceps femoris among the four treatments.

2) In the four methods, there was no obvious difference in the neuron efficiency of rectus femoris, vastus medialis muscle, vastus lateralis muscle or quadriceps femoris among the four treatments between the dominant leg and non-dominant leg.

3) The neuron efficiency of rectus femoris, vastus medialis muscle, vastus lateralis muscle or quadriceps femoris showed the same varying pattern, namely gradually decreasing from (a) to (d).

Table 3. Four muscles nerve efficiency characteristics Comparison between different vibration modes $(\mu \mathrm{v} / \mathrm{kg})$

\begin{tabular}{llllll}
\hline & & Rectus femoris & Vastus intermedius & Vastus lateralis & Quadriceps \\
\hline HFHA(a) & Dominant & $62.58 \pm 8.14$ & $71.45 \pm 6.15$ & $78.77 \pm 8.69$ & $212.29 \pm 15.14$ \\
& Non-Dominant & $63.17 \pm 7.58$ & $72.02 \pm 8.77$ & $77.25 \pm 6.25$ & $210.12 \pm 16.25$ \\
LFLA(b) & Dominant & $61.15 \pm 8.84$ & $70.26 \pm 6.15$ & $76.21 \pm 9.02$ & $208.17 \pm 11.77$ \\
& Non-Dominant & $61.89 \pm 5.47$ & $71.21 \pm 9.14$ & $76.59 \pm 4.36$ & $207.23 \pm 12.36$ \\
HFLA(c) & Dominant & $60.35 \pm 6.14$ & $69.58 \pm 8.16$ & $75.66 \pm 6.27$ & $203.15 \pm 31.22$ \\
& Non-Dominant & $60.14 \pm 8.01$ & $69.86 \pm 5.69$ & $75.15 \pm 8.02$ & $200.65 \pm 12.98$ \\
CON (d) & Dominant & $59.25 \pm 6.57$ & $68.53 \pm 5.47$ & $74.18 \pm 7.07$ & $197.11 \pm 12.36$ \\
& Non-dominant & $58.88 \pm 5.69$ & $68.15 \pm 6.23$ & $75.01 \pm 6.12$ & $199.03 \pm 11.25$ \\
& Between dominants & Pab,Pac,Pad & Pab, Pac, Pad, Pbc, & Pab,Pac,Pad ,Pb Pab, Pac, Pad, Pbc, \\
LSD & & Pbc, Pbd, Pcd & Pbd,Pcd & c, Pbd, Pcd & Pbd, Pcd \\
\cline { 2 - 6 } comparison & Between & Pab,Pac,Pad ,Pb & Pab, Pac, Pad, Pbc, & Pab, Pac, Pad, Pab, Pac, Pad, Pbc, \\
& Non-dominants & $c, P b d, P c d$ & Pbd, Pcd & Pbc, Pbd, Pcd & Pbd, Pcd \\
\hline Discd
\end{tabular}

\section{Discussion}

The 30sWingate test showed that there was no obvious difference in peak power and relative peak power occurring in the 8 subjects. The reasons for this might be as follows:

Firstly, vibration intensity might be insufficient. Mechanical vibration could cause reflexible contraction and further cause Tonic vibration reflex (TVR). Such reflex could gather more motor units through monosynapse or polysynapse. Insufficient vibration intensity might fail to cause the excitement of a motor neuron, which would impact the reflex mechanism of TVR. Vibration frequency of this study was $30 \mathrm{HZ}$, the same with that adopted in Cardinale study. But the result was different. Was this possibly related to low amplitude? We found in our trial test that human body might feel uncomfortable when the vibration frequency was between $25-30 \mathrm{HZ}$ while vibration acceleration exceeded $2.5 \mathrm{~g}$ and duration of vibration exceeded $12 \mathrm{~min}$. Thus we set the acceleration of this study at $2 \mathrm{~g}$ which might be slightly lower 
than that of other studies. Bosco found that 5.4g vibration intensity could markedly improve single-leg drive. The study of Cormic (2003) showed that $7 \mathrm{~g}$ and $9 \mathrm{~g}$ vibration intensity could improve jumping squat performance. Thus further proof was needed to confirm whether vibration intensity increase could improve sport performance.

Secondly, the anaerobic test duration might be too long. Previous studies showed that single vibration could markedly improve the MVC of wrist extensor within 5s (Curry, E. L., \& C1elland, J. A.1981), MVC of knee extensor within 5s

(Humphries, B. et al., 2004), the height of jumping squat (Cochrane, D.J.,\& Stannard, S.R., 2005), and the explosive force of upper limbs (Issurin, V. B. \& Tenenbaum, G.,1999), but could not improve 1 min. MVC of instep flexor. Therefore, 30s anaerobic test after vibration might not be able to improve sport performance.

The study found that the EMGrms of vastus lateral muscle and quadriceps femoris of the dominant leg under HFLA treatment was much higher than the other three. Besides, the EMGrms of rectus femoris of the non-dominant leg under HFLA treatment was much higher than that of HFHA, indicating that the quadriceps femoris of the non-dominant leg might gather more motor units. Interestingly, however, there was no markedly difference in peak power and average power among different methods. In other words, the increase of motor units might result from muscle fatigue. Some researchers believe that this might be because that the consumption of chemical transmitter between cynapse exceeded the supply.

What was strange was the inconsistent myoelectricity activity result between the dominant leg and non-dominant leg. Zoladz et al. (2007) measured the energy substance density of the dominant leg and non-dominant leg of players engaging in different sport event. The result showed that the ADP density of the non-dominant leg $(41.88 \mu \mathrm{m})$ was lower than that of the dominant leg $(65.61 \mu \mathrm{m})$ when players stayed calm. A 30s anaerobic exercise could consume a great amount of ATP and CP. The dramatic decrease of ATP could result in the increase of ADP density, which could activate glycolysis system and compound more ATP. In case of low ADP density, ability of the non-dominant leg to compound ATP through glycolysis system might not be as good as the dominant leg. Thus the non-dominant leg got fatigue earlier and generated obvious myoelectricity active reaction. Besides, Jacobs found that the peak moment of force of hip abductors of the non-dominant leg was much lower than that of the dominant leg. Heuer (2007) pointed out that feedforward control and movement accuracy of the dominant leg was superior to that of the non-dominant leg. Therefore, the increased motor unit and the further increased EMG might be because of the difference of neural control system and the necessity for the low moment of force of the non-dominant leg to match with the power output of the dominant leg. Yet further study was needed to verify whether the myoelectricity activity of the dominant leg and non-dominant leg of players engaging in other sport events was similar to that of long jumpers.

There was no obvious difference in rectus femoris, vastus lateral muscle, vastus medialis or quadriceps femoris among the four methods. The increase of advanced motor center activity could improve the neuromuscular adaptation. Such adaptation had the same effect with the marked increase of leg extensor through resistance training. Therefore, Bosco (1999) believed that whole-body vibration could improve neural efficiency. But the result of this study did not show obvious improvement in neural efficiency. This might be related to insufficient vibration intensity and prolonged test time. The vibration acceleration (vibration intensity) in Bosco's study was much higher than this study. Therefore, vibration intensity might be the determinant. Some data of this study might support this assumption. The mean value of HFHA was lower than that of LFLA and HFLA (though not obvious), indicating that high frequency and high amplitude vibration could potentially impact neural efficiency.

Besides insufficient vibration intensity, another reason for the unobviousness was that the vibration duration was too short and the motor nervous system could not adapt to the external stimulation within such short period of time. Rehn (2007) pointed out that there was no consistent positive result with short-period vibration stimulation. Long-term whole-body vibration had a better effect on muscle performance improvement. Therefore, long-term whole-body vibration training seemed to be the proper strategy to improve players' muscle force and explosive power.

\section{Conclusion}

For long jumpers, the peak power and relative peak power of their dominant leg were superior to that of the non-dominant leg. The anaerobic power descent rate of the dominant leg was much lower than that of the non-dominant leg. But such anaerobic performance seemed to be immune to single vibration training. The time to reach the maximum anaerobic power was different in the four methods. HFHA needed the least time; then was the HFLA; and LFLA was the last. Under HFHA and HFLA, the dominant leg needed less time than the non-dominant leg.

Rectus femoris EMGrms of the dominant leg was not impacted by vibration methods. For the non-dominant leg, LFLA had the strongest rectus femoris activity and then were HFHA and HFLA. Besides, the rectus femoris activation level of the non-dominant leg was higher than that of the dominant leg under the three methods. EMGrms\% of vastus medialis of the dominant leg had the strongest activity under HFHA and LFLA. Under LFLA, the activation level of the dominant leg was higher than its counterpart. Vastus lateral muscle EMGrms\% of the dominant leg had the strongest 
activity under LFLA and HFLA while that of the non-dominant leg had the strongest activity under HFLA. Under LFLA and CON (d), the activation level of the dominant leg was significantly higher than that of the non-dominant leg.

Under HFLA, the myoelectricity activity of the dominant leg was significantly higher than the other three methods. The non-dominant leg had the strongest activity under LFLA and HFLA. Besides, the quadriceps femoris activation level of the dominant leg was much higher than that of the non-dominant leg under these two methods.

Whether for the dominant or non-dominant leg, the neural efficiency of rectus femoris, vastus medialis muscle, vastus lateral muscle or quadriceps femoris was not impacted by vibration methods. But the neural efficiency decreased gradually in order of HFHA, LFLA, HFLA, CON, seemingly indicating that high frequency high amplitude vibration could potentially impact neural efficiency.

\section{Acknowledgement}

This study was supported by Chongqing Administration of Sport (No. B201405).

\section{References}

Bosco, C. ,Cardinnale, M., \& Tsarpe1a, O. (1999). Influence of of vibration on mechanica1 power and electromyogram activity in human arm flexor muscles[J]. European Journal of Applied Physiology 79(4), 306-311. http://dx.doi.org/10.1007/s004210050512

Bosco, C., Colli, R., Introini, E., Tsarpela, O., \& Madella, A. et al. (1999). Adaptive responses of human skeletal muscle to vibration exposure. Clinical Physiology, 19(2), 183-187. http://dx.doi.org/10.1046/ j.1365-2281.1999.00155.x

Bosco, C., Iacovelli, M., Tsarpe1a, O., Bonifazi, M., \& Tihanyi, J. et al. (2000). Hormonal responses to who1e-body vibration in men. European Journal of Applied Physiology 81(6), 449-454. http://dx.doi.org/10.1007/s004210050067

Cardinale, M., \& Bosco, C. (2003). The use of vibration as an exercise intervention. Exercise and Sport Sciences Review, 31(1), 3-7. http://dx.doi.org/10.1097/00003677-200301000-00002

Cochrane, D. J., \& Stannard, S. R. (2005). Acute whole body vibration training increases vertica1 jump and flexibility performance in elite fema1e fie1d hockey p1ayers. British Journal of Sports Medicine. 39(11), 860-865. http://dx.doi.org/10.1136/bjsm.2005.019950

Cormie, P., Deane, R. S.,Triplett, N. T., \& McBride, J. M. (2006). Acute effects of whole-body vibration on muscle activity, strength and power. Journal of Strength and conditioning Research, 20(2), 257-261. http://dx.doi.org/10.1519/r-17835.1

Curry, E. L., \& C1elland, J. A. (1981). Effects of the asymmetric tonic neck reflex and high-frequency muscle vibration on isometric wrist extension strength in norma1 adults. Physical Therapy, 61(4), 487-495.

Heuer, H. (2006). Contro1 of the dominant and nondominant hand: exploitation and taming of nonmuscular forces. Experimental Brain Research, 178(3), 363-373. http://dx.doi.org/10.1007/s00221-006-0747-5

Humphries, B.,Warman, G., Purton, J., Doyle, T., \& Dugan, E. (2004). The influence of vibration on muscle activation and rate of force development during maxima1 isometric contractions. Journal of Sports Sciences and Medicine, $3(1), 16-22$.

Ingham, S. A., Whyte, G. P., \& Nevill, A . M. (2002). Determinants of $2000 \mathrm{~m}$ rowing ergometer performance in elite rowers. European Journal of Applied Physiology, 88(3), 243-246. http://dx.doi.org/10.1007/s00421-002-0699-9

Issurin, V. B., \& Tenenbaum, G. (1999). Acute and residual effect of vibratory stimu1ation on explosive strength in elite and amateur athletes. Journal of Sports Sciences, 17(3), 177-182. http://dx.doi.org/10.1080/026404199366073

Jacobs , C., Uh1, T. L., See1ey, M., Sterling, w., \& Goodrich, L. (2005). Strength and fatigability of the dominant and nondominant hip abductors. Journal of Athletic Training, 40(3), 203-206.

Rehn, B., Lidstrom, J., Skoglund, J., \& Lindstrom, B. (2006). Effects on leg muscular whole-body vibration exercise: a systematic review. Scandinavian Journal of Medicine and Science in Sports. 17(1), 2-11. http://dx.doi.org/10.1111/j.1600-0838.2006.00578.x

Zoladz, J. A., Zapart-Bukowska, J., Majerczark J., \& Korzeniewskim, B. et al. (2007). Phosphorylation potential in the dominant leg is lower, and ADP-free is higher in calf muscles at rest in endurance athletes than in sprinters and in untrained subjects. Journal of Physiology and Pharmacology, 58(4), 803-819.

This work is licensed under a Creative Commons Attribution 3.0 License. 